\title{
MRI BRAIN IMAGE SEGMENTATIN AND CLASSIFICATION BY MODIFIED FCM \&SVM AKORITHM
}

\author{
G.B.Deshmukh, P.D.Lambhate \\ ${ }^{1}$ M.E. Student, Computer Engineering, JSCOE, Maharashtra, India, gopaldeshmukh16@yahoo.in \\ ${ }^{2}$ Asso Professor, Information Technology, JSCOE, Maharashtra, India,pinu_poonam@yahoo.co.in
}

\begin{abstract}
Brain Tumor detection is challenging task in biomedical field. Image segmentation is a key step from the image processing to image analysis, it occupy an important place. The manual segmentation of brain image is challenging and time consuming task. An automated system overcomes the drawbacks as well as it segments the white matter, grey matter, cerebrospinal fluid and edema. This clustering approach is particularly used for brain tumor detection in abnormal MR images. In this paper the application of Modified FCM algorithm for Brain tumor detection and its classification by SVM algorithm is focused. The Magnetic Resonance image is converted in to vector format and that is given as input to the modified fuzzy c-means algorithm. In modified fuzzy c-means the steps are: initial fuzzy partitioning and fuzzy membership generation Cluster updation based on objective function, Assigning labels to pixels of each category and display segmented image that will give more meaningful regions to analyze. This clustered images served as inputs to SVM. The basic SVM takes a set of input data and predicts, for each given input, which of two possible classes.
\end{abstract}

Keywords: Clustering, Classification, Fuzz C-Means, Support Vector Machine, MRI, Brain Tumor.

\section{INTRODUCTION}

The medical field is very vast and challenging. Medical image analysis has great importance especially in noninvasive treatment and study of deceases. Analysis tool helps both doctor and radiologist to arrive at specific diagnosis of disorder. Medical image data is obtained form magnetic resonance immerging (MRI), Computed Tomography(CT) etc which indicates the presence or absence of lesion along with patient history. The segmentation technique mostly preferred by Radiologist to segment input medical image intends the size of tumor can be tracked using this technique which helps for treatment planning. Previously manual segmentations used which is time consuming and prone to human errors. Challenges in Automatic Brain MR Image .

\subsection{Tumor Segmentation}

One of the most prominent issues in brain tumor segmentation is that tumor pixels could have similar or identical signal intensity in comparison to normal pixels even within the same image. An additional challenge is that tumor areas often have heterogeneous intensities. Furthermore, the large variation of properties amongst different tumor types makes the task of distinguishing tumor from normal brain tissues more complex. Meaningful regions. The abnormal MR image is segmented so as to detect Tumor. The specific area Detection of brain tumor can be done with the help of segmentation but to classify its type is challenging process. This need motivates to combine both clustering and classification together. Image segmentation can be performed effectively by clustering image pixels. Modified Fuzzy C-Means is used to clustering and Support Vector machine algorithm is used for classification.

\section{RELATED WORK}

A researcher in the field of biomedical engineering not only needs to be familiar with the relevant applications of engineering in medicine but also with the basic life sciences [1].This interaction between the traditional engineering field and modern medicine is for us the motivation to tackle this paper. It is interesting to see how new techniques in the field of engineering can improve medical diagnosis and health care. Many techniques have been reported for clustering and classification of brain tumor in MR images. Clustering is one of the widely used segmentation technique. Fuzzy CMeans algorithm is mostly preferred due to its flexibility .But major operational drawback is that it is time consuming [2]. A hierarchical FCM algorithm uses the concept of template matching proposed by Kwon and Han[4].But has drawback of requirement of accurate emplate.FCM is also implemented by parallel processing [3] but hardware implementation is not effective it will not achieve desired efficiency. Cheng and Goldgof [4] proposed the fast clustering algorithm based on random sampling. Fast fuzzy clustering for web documentation which is highly robust is proposed in [5] .Modified Fuzzy C-Means algorithm uses the technique of data compression for clustering [6].But has the drawback it is not able to specify the class of tumor. Many techniques have been reported for classification of 
brain tumors in MR images, most notably, support vector machine (SVM) [7] neural network [8], knowledge based techniques [9].Classification rate of Neural Network is lower than SVM Chaplot etal [10].SVM it is an very powerful method of obtaining model for classification [11].SVM provides a mechanism for choosing model structure in which it gives low generalizationrisk.SVM produces output which is combination of the training example projected on high dimensional feature space through the use of kernel space. been reported for clustering and classification of brain tumor in MR images. Clustering is one of the widely used segmentation technique. Fuzzy CMeans algorithm is mostly preferred due to its flexibility .But major operational drawback is that it is time consuming [2]. A hierarchical FCM algorithm uses the concept of template matching proposed by Kwon and Han[4].But has drawback of requirement of accurate template.FCM is also implemented by parallel processing [3] but hardware implementation is not effective it will not achieve desired efficiency. Cheng and Goldgof [4] proposed the fast clustering algorithm based on random sampling. Fast Fuzzy clustering for web documentation which is highly robust is proposed in [5] .Modified Fuzzy C-Means algorithm uses the technique of data compression for clustering [6].But has the drawback it is not able to specify the class of tumor. Many techniques have been reported for classification of brain tumors in MR images, most notably, support vector machine (SVM) [7] neural network [8], knowledge based techniques [9].Classification rate of Neural Network is lower than SVM Chaplot etal [10].SVM it is an very powerful method of obtaining model for classification [11].SVM provides a mechanism for choosing model structure in which it gives low generalizationrisk.SVM produces output which is combination of the training example projected on high dimensional feature space through the use of kernel space.

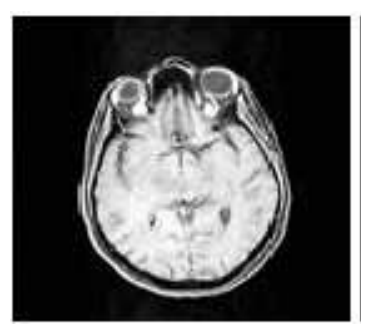

Fig -1: MRI Image1

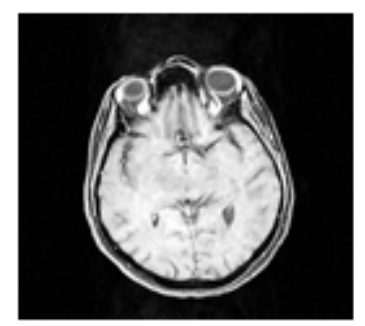

Fig-2:MRI Image2
In our proposed method, we have to pre-process the given test image for reducing noise and to enhance the contrast. Then, we have to segment the brain image using modified fuzzy c-means algorithm to visually show the normality's such as tumor. Afterwards, texture features (GLCM) will be extracted from it. In feature extraction stage, statistical measurements are calculated from the gray level cooccurrence matrix for different directions and distances. Among the various features extracted. We have to select the distinct features that will be utilized for classification purpose. For the selection of features SFS(Sequential Forward Selection) is used. Support vector machine (SVM) is used to classify whether the test image comes under normal, benign and malignant.

\subsection{Steps For Tumor Classification}

1. Preprocessing (Median filter)

2. Segmentation (Modified FCM)

3. Feature extraction (GLCM technique)

4. Feature selection (Sequential forward selection)

5. Classification by SVM

1. Preprocessing (Median filter)

\section{Median Filtering:}

Median filtering operation, the pixel values in the neighborhood window are ranked according to intensity, and the middle value (the median) becomes the output value for the pixel under evaluation. Median filtering does not shift boundaries, as can happen with conventional smoothing filters. Since the median is less sensitive than the mean to extreme values (outliers), those extreme values are more effectively removed. Median filtering preserves the edges.

\section{Segmentation (Modified FCM)}

First the conventional FCM was analyzed. The results show that it does not produce a standard segmentation result always due to the random nature of initial membership values. This is the main drawback in conventional FCM. In this phase modified fuzzy c-means segmentation is implemented to overcome this problem by initializing standard membership values (computed by template averaging) so that standard segmentation result for each and every image was obtained. The number of clusters, initial standard fuzzy membership values and the feature vector were given as the input. After the completion of initial clustering process, the clusters were updated in turn updates the cluster weights and membership degree. An objective function will determine that up to which the clusters should be updated. Vector membership values are distributed identically to all members in that cluster. The convergence rate is highly improved when compared with the conventional FCM. Finally the intra cluster similarity was computed to determine which cluster is having high similarity among its members. In addition to that the algorithm was executed for various numbers of clusters for the computation of Davies Bouldin index. This index was useful in the prediction of correct number of clusters to be given in advance. Fuzzy partitioning is carried out through

\section{PROPOSED WORK}


an iterative procedure that updates membership uij and the cluster centroids cj by

$$
\begin{aligned}
u_{i j} & =\frac{1}{\sum_{k=1}^{C}\left(\frac{\left\|x_{i}-c_{j}\right\|}{\left\|x_{i}-c_{k}\right\|}\right)^{\frac{2}{m-1}}} \\
c_{j} & =\frac{\sum_{i=1}^{N} u_{i j}^{m} x_{i}}{\sum_{i=1}^{N} u_{i j}^{m}}
\end{aligned}
$$

where $m>1$, and represents the degree of fuzziness (typically=2)

\section{Feature extraction (GLCM technique)}

Extraction of Text Feature:-

Text features are extracted by using Gray Level Cooccurrence Metrics Technique. A GLCM Pd[i,j] is defined by first specifying a displacement vector $\mathbf{d}=(\mathrm{dx}, \mathrm{dy})$ and counting all pairs of pixels separated by $\mathbf{d}$ having gray levels $\mathrm{i}$ and $\mathrm{j}$.

The GLCM is defined by:- where $\mathbf{n}_{\mathbf{i j}}$ is the number of occurrences of the pixel values $(\mathbf{i}, \mathbf{j})$ lying at distance $\mathbf{d}$ in the image.

The co-occurrence matrix $\mathbf{P}_{\mathbf{d}}$ has dimension $\mathbf{n} \times \mathbf{n}$, where $\mathrm{n}$ is the number of gray levels in the image.

\section{Feature selection (Sequential forward selection)}

Automatic feature selection is an optimization technique that, given a set of $m$ features, attempts to select a subset of size $\boldsymbol{n}$ that leads to the maximization of some criterion function. Feature selection algorithms are important to recognition and classification systems because, if a feature space with a large dimension is used, the performance of the classifier will decrease with respect to execution time and to recognition rate. The execution time increases with the number of features because of the measurement cost. The recognition rate can decrease because of redundant features and of the fact that small number of features can alleviate the course of dimensionality when the training samples set is limited, leading to overtraining. On the other hand, a reduction in the number of features may lead to a loss in the discrimination power and thereby lower the accuracy of the recognition system. In order to determine the best feature subset for some criterion, some automatic feature selection algorithm can be applied to the complete feature space, varying the number of selected features from 1 to $m$.
1) Training/Clustering Stage: the process of defining criteria by which patterns are recognized, developing a numerical description for each class.

2) Classification Stage: each pixel in the image data set is categorized into the class it most closely resembles based on a mathematical decision rule

3) Output Stage: results are presented in a variety of forms (tables, graphics, etc.)

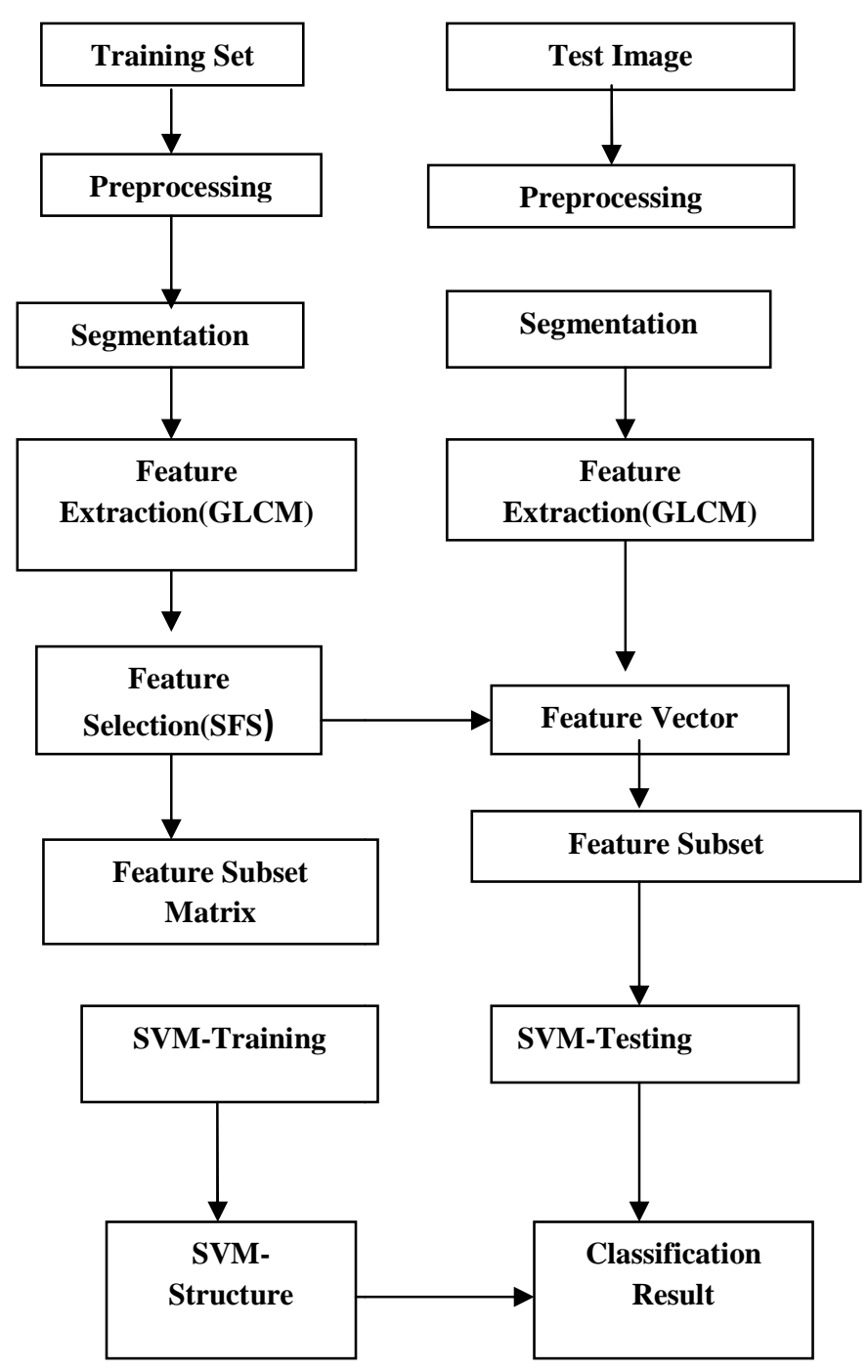

Fig 3: Proposed Design

\section{CONCLUSION}

The work in this paper involves Modified Fuzzy CMeans for segmentation the input which is MR Image to detection of abnormality from brain and SVM to classify the input from modified FCM to detect the type of tumor. The intention of the paper is to get More accurate classification of tumor into four types.

\section{REFERENCES}

\section{Classification by SVM}


[1]Breitenmoser,"Evalutation and implementation of neural brain activity detection methods for fMRI", Linkoping University, Sweden,21 February 2005,pp.13-18.

[2] Jie Yu, Peihuang Guo, Pinxiang Chen, Zhongshan Zhang and Wenbin Ruan, "Remote sensing image classification based on improved fuzzy c-means", Geo-Spatial Information Science,IEEE transactionsvo1.11, no.2, pp:9094, 2008.

[3] S.Murugavalli and V.Rajamani, "A high speed parallelfuzzy Cmean algorithm for brain tumor segmentation", BIME journal,IEEE transactions vo1.6, no.1, 2006

[4] Tai Wai Cheng, Goldgof and Lawrence Hall, "Fast fuzzy clustering", Fuzzy Sets and Systems,IEEE transactions vo1.93, pp:49-56, 1998.

[5] R Krishnapmam and Y Li, "A fuzzy relative of the kmedoids algorithm with application to web document and snippet clustering", IF-EE nternationalFuzzy Systems conference, vo1.3 pp:1281-1286, 1999.

[6] M. Shasidhar, V.Sudheer Raja, B. jayKkumarmri brain image segmentation using modified fuzzy cmeans clustering algorithm , 978-0-7695-4437-3/11 , 2011 IEEE.

[7] Mohd Fauzi Bin Othman, Noramalina, Bt Abdullah, Nurul Fazrena, Bt Kamal MRI Brain classification using Support vector machine 978-1-4577-0005- 7/11/,2011 IEEE. [8] Shan Shen, William Sandham, Member, IEEE, Malcolm Granat, and Annette Sterr mri fuzzy segmentation of brain tissue using neighborhood attractionwith neural-network optimization IEEE transactions on information technology in biomedicine, vol. 9, no. 3, september 2005

[9] Clark M.C., Hall L.O., Goldgof D.B., Velthuizen R., Murtagh F.R. and Silbiger M.S.Automatic tumor segmentation using knowledge based techniques, IEEE Transactions on Medical Imaging, 1998, 17(2),p. 187192.[10] Chaplot S., Patnaik L.M., Jagannathan N.R., Classificationof magnetic resonance brain imagesusing wavelets as input to support vector machine and neural network, Biomedical SignalProcessing and Control, 2006, 1(1), p. 86-92.7

[11] C. J. C. Burges, "A tutorial on support vector machines for pattern recognition", Data Mining and Knowledge Discovery, 2(2):121-167, 1998.

[12] Shan Shen, William Sandham, Member, IEEE, Malcolm Granat, and Annette Sterr IEEE transactions on information technology in biomedicine, vol. 9, no. 3, September 2005

[13] S R Kannan, "Segmentation of MRI using new unsupervised fuzzy C-means algorithm", ICGST-GVIP journal, vo1.5, no.2, 2005.

[14] S.Murugavalli and V.Rajamani, "A high speed parallel fuzzy Cmean algorithm for brain tumor segmentation", BlME journal, vo1.6, no.1, 2006.8
G.B.Deshmukh is M E Computer student and currently doing work on data mining (classification and clustering of MRI Brain Image for Tumor.)

P.D.Lambhate is Associate professor and Head of the department from Information Technology.

\section{BIOGRAPHIES}

